\title{
AUFSATZ
}

\section{Das Scheitern des Spitzenkandidatensystems und andere populäre Irrtümer über die Demokratisierung der Europäischen Union}

Frank Decker

Der Machtpoker um das Amt des Kommissionspräsidenten, der sich zwischen Europäischem Parlament (EP) und Rat nach der Europawahl abgespielt hat, bestätigt die grundsätzlichen Zweifel am sogenannten Spitzenkandidatensystem. Die Hoffnungen, dass mit ihm bei den Wahlen zum EP „mehr Demokratie“ einzieht und die Bürger näher an die europäischen Institutionen herangeführt werden, haben sich nicht erfüllt. Warum ist das so? In der Debatte um die Bestellung des Kommissionspräsidenten lassen sich viele populäre Irrtümer ausmachen, die von Befürwortern und Skeptikern einer demokratischen Weiterentwicklung ${ }^{1}$ der europäischen Institutionen gleichermaßen kolportiert werden. Die Missverständnisse zeugen einerseits von einem fehlenden Verständnis der Dynamik historischer Demokratisierungsprozesse. Zum anderen rühren sie daher, dass man die demokratischen Standards oder Defizite der EU einseitig am Maßstab der nationalstaatlichen Regierungssysteme misst. Dies gilt für die Demokratie im Allgemeinen, die an bestimmte Voraussetzungen wie eine gemeinsame Öffentlichkeit oder ein (staats)bürgerschaftliches Verbundenheitsgefühl geknüpft ist, die in der EU erst ansatzweise vorliegen. Und es gilt mit Blick auf die institutionelle Gestalt des EU-Regierungssystems im Besonderen, die der - mit Ausnahme Zyperns in allen EU-Mitgliedstaaten gegebenen ${ }^{2}$ - parlamentarischen Regierungsform nur bedingt entspricht.

\section{Hat die Europäische Union ein parlamentarisches Regierungssystem?}

Der erste - von den Skeptikern der Demokratisierung begangene - Irrtum besteht im Verweis darauf, dass das Spitzenkandidatensystem nirgendwo festgeschrieben sei. Das ist für sich betrachtet richtig, führt aber am Kern des Problems vorbei. Denn zum Wesen jeder verfassungsstaatlichen Demokratie gehört, dass sie neben den in der Verfassung und weiteren Gesetzen kodifizierten Normen ungeschriebene Regeln kennt, die für den Charakter des Regierungssystems maßgebliche Bedeutung erlangen. Auch die Verfassungsvorschriften selbst lassen sich häufig nur in Verbindung mit der tatsächlichen Praxis verstehen, die sie durch diese Regeln entfalten. Nirgendwo zeigt sich das deutlicher als bei der Frage, auf

1 Gleichbedeutend damit steht der im Folgenden verwandte Begriff der „Demokratisierung“. Zu dessen Implikationen im Kontext der EU vgl. Frank Decker, Demokratie und Demokratisierung jenseits des Nationalstaats. Das Beispiel der Europäischen Union, in: ZPol, 10. Jg. (2000), H. 2, S. $585-629$.

2 Außerhalb der EU weist unter den konsolidierten demokratischen Staaten in Europa lediglich die Schweiz ein nicht-parlamentarisches Regierungssystem auf. Vgl. Winfried Steffani, Art. „Parlamentarisches und präsidentielles Regierungssystem“, in: Manfred G. Schmidt (Hrsg.), Die westlichen Länder, München 1992 (Lexikon der Politik, Band 3, hrsg. von Dieter Nohlen), S. 292 - 293. 
welche Weise die Regierung beziehungsweise der Regierungschef bestellt wird, die jetzt nach 2014 bereits zum zweiten Mal die europäischen Gemüter erhitzt hat.

Was das Europäische Parlament 2014 vermochte und 2019 nicht vermochte - dem Europäischen Rat die Bestellung des Kommissionspräsidenten streitig zu machen - hat sein Vorbild in der Genese des parlamentarischen Regierungssystems. Dessen Ursprungsort liegt bekanntlich in Großbritannien. Beginnend mit der Glorious Revolution Ende des 17. Jahrhunderts war es dem Parlament dort gelungen, dem König das Recht, „seine“ Regierung selbst zu bestimmen und zu entlassen, frühzeitig zu entwinden. Gerade deshalb konnte die Monarchie im Vereinigten Königreich als politisch machtlose Institution überleben, während sie in den verspäteten Demokratien Frankreichs, Deutschlands oder Italiens durch ein gewähltes Staatsoberhaupt ersetzt wurde. ${ }^{3}$ Für alle nationalen parlamentarischen Regierungssysteme ist charakteristisch, dass sie die Staatsoberhäupter an der Bestellung und Entlassung der Regierung förmlich beteiligen. Die in den meisten Verfassungen gewählte Formulierung, wonach der Monarch beziehungsweise Präsident den Regierungschef „ernennt“ oder diesen dem Parlament zur Wahl „vorschlägt“ (wie in der Bundesrepublik), täuscht jedoch darüber hinweg, dass er dabei in seiner Entscheidung nicht frei ist, sondern an den Willen der Abgeordneten gebunden bleibt. Genau das schwebt den Verfechtern einer parlamentarischen Demokratisierungsstrategie für die EU vor.

An dieser Stelle kommt ein zweiter Irrtum ins Spiel, diesmal von den Befürwortern des Spitzenkandidatensystems. Sie übersehen, dass das eigentliche Kernmerkmal des parlamentarischen Systems nicht die positive Bestellung oder förmliche Wahl der Regierung beziehungsweise des Regierungschefs durch das Parlament ist, sondern ihre jederzeit mögliche Abberufung. . ${ }^{4}$ Diese zieht das Bestellungsmerkmal „funktionslogisch“ nach sich, denn es wäre widersinnig, wenn das Staatsoberhaupt eine Person ernennt, von der es weiß oder wissen müsste, dass das Parlament sie bereits am nächsten Tag wieder abwählt. Der spezielle Charakter des EU-Regierungssystems zeigt sich darin, dass es ein reguläres Misstrauensvotum nicht kennt. Der Kommissionspräsident wird vom Parlament mit absoluter Mehrheit gewählt; seine Abberufung, die gleichbedeutend mit der Abberufung der gesamten Kommission ist, setzt dagegen eine Zweidrittelmehrheit voraus. Dieses Verfahren ähnelt eher dem US-amerikanischen „impeachment“, auch wenn es anders als dort nicht an „hohe Verbrechen oder Vergehen " geknüpft ist. ${ }^{5}$ Dem entspricht das Nichtvorhandensein einer vorzeitigen Parlamentsauflösung in der EU, das in einem normalen parlamentarischen System das Pendant zum Misstrauensvotum bildet. Damit soll dafür Sorge getragen werden, dass beim Verlust der parlamentarischen Mehrheit der Regierung jederzeit eine neue Mehrheit erzeugt werden kann. ${ }^{6}$

3 Vgl. Giovanni Sartori, Comparative Constitutional Engineering: An Inquiry into Structures, Incentives and Outcomes, Houndmills u.a. 1997, S. 85.

4 Vgl. Winfried Steffani, a.a.O. (Fn. 2).

5 Laut Art. 234 des Vertrags über die Arbeitsweise der Europäischen Union (AEUV) kann ein Misstrauensantrag aber nicht aus allgemeinen politischen Gründen, sondern nur „wegen der Tätigkeit“ der Kommission eingebracht werden. Dabei geht es vorrangig um Verletzungen ihrer Amtspflichten, also zum Beispiel Missmanagement oder Korruption. Vgl. Jared Sonnicksen, Ein Präsident für Europa. Zur Demokratisierung der Europäischen Union, Wiesbaden 2014, S. 131.

6 Vgl. Frank Decker / Jared Sonnicksen, Noch auf dem Pfad zu einem präsidentiellen System? Eine Analyse der horizontalen Gewaltenteilung der EU-Polity nach der Europawahl 2014, in: Miriam Hartlapp / Claudia Wiesner (Hrsg.), Gewaltenteilung und Demokratie im Mehrebenensystem der EU, ZPol-Sonderheft 1/2016, Wiesbaden 2016, S. $71-84$. 
Diese „Abweichungen“ erklären, warum der Parlamentarismus in der EU anders funktioniert als in den nationalstaatlichen Demokratien. Während es in den dortigen parlamentarischen Systemen ein festgefügtes Gegenüber von regierungstragender Mehrheit und Opposition gibt, bilden sich bei den Gesetzesbeschlüssen im Europäischen Parlament - je nach Materie - unterschiedliche Abstimmungskoalitionen heraus; regiert wird mit „wechselnden Mehrheiten“. Auch innerhalb der Fraktionen ist das Abstimmungsverhalten häufig uneinheitlich, was auf deren heterogene Zusammensetzung und die fortbestehenden nationalen Prägungen der Fraktionsmitglieder verweist. Dem Europäischen Parlament mögen zwar bis heute ein eigenes Initiativrecht oder die volle gesetzgeberische Gleichstellung mit dem Rat fehlen. Wo es mitentscheidet, übt es de facto aber größeren Einfluss aus als die Parlamente in den Mitgliedstaaten, die die Funktion der Gesetzgebung weitgehend an die jeweiligen Regierungen abgetreten haben.

\section{Das Problem der fehlenden Stimmrechtsgleichheit}

Der populäre Irrtum, wonach das EP ein „schwaches“ Parlament sei, lässt sich daher entkräften. Schwerer fällt dies bei einem anderen Argument, das von Skeptikern der Demokratisierung wie dem Berliner Historiker Heinrich August Winkler ${ }^{7}$ regelmäßig vorgebracht wird und auch in der Judikatur des Bundesverfassungsgerichts eine wichtige Rolle spielt, um der Straßburger Versammlung die Eigenschaft eines „richtigen“ Parlaments abzusprechen: der Verstoß gegen das Prinzip der demokratischen Wahlrechtsgleichheit. Die Sitzkontingente, die den Mitgliedsländern im EP zugeteilt werden, orientieren sich - wie man weiß - nicht streng an deren Bevölkerungsgröße, sondern entsprechen dem Prinzip der „degressiven Proportionalität“: Kleinere Länder werden so gegenüber den großen begünstigt. Dies hat zum Beispiel zur Folge, dass in Deutschland für ein Mandat elfmal so viele Stimmen benötigt werden wie in Malta. Will man den kleineren Ländern eine faire Vertretungschance belassen, ist eine solche Verzerrung kaum zu vermeiden, es sei denn, man würde das mit 751 Abgeordneten ohnehin schon sehr große Parlament noch weiter vergrößern.

Darin einen grundlegenden Verstoß gegen die Demokratie zu sehen, erscheint jedoch aus zwei Gründen zu puristisch: Erstens wird die ungleiche Repräsentation im Europäischen Parlament teilweise dadurch ausgeglichen, dass die unterschiedlichen Bevölkerungsgrößen auch in den Abstimmungsregeln des Rates Berücksichtigung finden (durch das Prinzip der „doppelten Mehrheit“). Die EU folgt also nicht dem Modell klassischer Zweikammersysteme wie die USA oder die Schweiz, wo die eine Kammer strikt nach dem demokratischen, die andere nach dem föderativen Gleichheitsprinzip zusammengesetzt ist, sondern „mischt" die Prinzipien in ihren beiden Kammern. ${ }^{8}$ Zweitens - und noch wichtiger - kommt es für die Gleichheit nicht primär auf die territoriale, sondern auf die (partei) politische Repräsentation an. Diese herzustellen wäre im Europäischen Parlament durch die

7 Vgl. Heinrich August Winkler, Ein Anspruch auf tönernen Füßen, in: FAZ vom 29. Juni 2019, S. 6.

8 Vgl. Jürgen Habermas, Zur Prinzipienkonkurrenz von Bürgergleichheit und Staatengleichheit im supranationalen Gemeinwesen. Eine Notiz aus Anlass der Frage nach der Legitimität der ungleichen Repräsentation, in: Der Staat, 53. Jg. (2014), H. 2, S. $167-192$. 
Einführung entsprechender Ausgleichsmandate relativ problemlos möglich, ohne dass man an der ungleichen territorialen Verteilung rütteln müsste. ${ }^{9}$ Dazu - aber auch nur dazu könnten zum Beispiel transnationale Listen dienen. ${ }^{10}$

Deren Befürworter verbinden mit ihnen allerdings einen viel weiter reichenden Anspruch. Sie seien der Schlüssel, um die bisherige nationale Orientierung der Europawahlkämpfe zu überwinden und eine wirkliche Europäisierung herbeizuführen. Dahinter verbergen sich mindestens zwei Irrtümer: Erstens ist die Verknüpfung von territorialer und (partei)politischer Repräsentation in den nationalstaatlichen Wahlsystemen nicht nur ein gängiges Prinzip. Sie ergibt auch Sinn, weil sich eine persönliche Beziehung der Wähler zu den Kandidaten ohne örtliche Nähe nicht herstellen lässt; und zweitens lenkt der Vorschlag von dem eigentlichen Grund der fehlenden oder unzureichenden Europäisierung ab. Er liegt darin, dass bei den Europawahlen bis heute keine europäischen, sondern nationale Parteien antreten - diese schließen sich erst nach den Wahlen im neu konstituierten Parlament zu gemeinsamen Fraktionen zusammen. Die beste Möglichkeit, das zu ändern, wäre die Einführung eines einheitlichen Wahlsystems mit einer europaweiten Zwei- oder Dreiprozenthürde. Eine solche Sperrklausel würde die Europäisierung des Parteiensystems erzwingen, weil dann allenfalls große Parteien aus großen Mitgliedstaaten noch aus eigener Kraft ins Parlament gelangen könnten. ${ }^{11}$

\section{Aporien des Spitzenkandidatensystems}

An dieser Stelle kommen die Spitzenkandidaten zum Tragen. Demokratie bedeutet, dass die Bürger in Wahlen über die Grundrichtung der Politik und das führende Regierungspersonal entscheiden können müssen. Dies setzt voraus, dass ihnen das Personal vorab bekannt ist und sie nicht die sprichwörtliche „Katze im Sack“ wählen. Es setzt nicht voraus, dass die Kandidaten überall förmlich zur Wahl stehen - ein weiterer populärer Irrtum. Auch Angela Merkel war bei der Bundestagswahl als Abgeordnete nur in Mecklenburg-Vorpommern (als Erstplatzierte der Landesliste) und Direktkandidatin im Wahlkreis Rügen wählbar. Theoretisch ist es noch nicht einmal nötig, dass die Spitzenkandidaten überhaupt als Abgeordnete

9 Behandelt man die Länder als abgeschlossene Wahlgebiete, wird das Problem der Gleichheit/ Ungleichheit allerdings durch die ungleiche Wahlbeteiligung verschärft. So machten bei der Europawahl 2014 in Belgien sieben Mal mehr Bürger von ihrem Wahlrecht Gebrauch als in der Slowakei (90 gegenüber 13 Prozent). Unabhängig vom Länderschlüssel wurden somit für ein belgisches Mandat sieben Mal so viele Stimmen benötigt wie für ein slowakisches. Dem ließe sich entgegentreten, wenn man die Sitzkontingente in Abhängigkeit von der Wahlbeteiligung in den einzelnen Ländern nach oben oder unten anpasst. Länder mit überdurchschnittlicher Wahlbeteiligung würden dann mehr, Länder mit unterdurchschnittlicher Beteiligung weniger Sitze erhalten. Länder mit einer Wahlpflicht (wie in Belgien oder Luxemburg) wären von dieser Regelung auszunehmen. Sie könnten ihre Sitzzahl also nur erhöhen, wenn sie auf die Wahlpflicht verzichten. Mit der Variation der Sitzkontingente hätten alle Länder einen starken Anreiz, für eine möglichst hohe Beteiligung in den nationalen Wahlgebieten zu sorgen.

10 Vgl. Manuel Müller, Europäische Zweitstimme: Für mehr Demokratie bei der Europawahl, in: dbb magazin, 68. Jg. (2017), H. 9, S. 26 - 27.

11 Vgl. Frank Decker, Weniger Konsens, mehr Wettbewerb: Ansatzpunkte einer institutionellen Reform, in: Jürgen Rüttgers / ders. (Hrsg.), Europas Ende, Europas Anfang, Bonn 2017, S. 163 180, S. $168 \mathrm{ff}$. 
kandidieren. In der Europäischen Union wären sie ohnehin gezwungen, ihr Mandat sogleich wieder abzugeben, wenn sie in die Kommission „aufrücken“, denn es gilt der Grundsatz der Inkompatibilität. Sinnvoll bleibt die Kandidatur potenzieller Kommissarsanwärter für das Europäische Parlament allemal. Einem Kandidaten die Eignung für den Chefposten in der EU abzusprechen, weil dieser „nur“ über legislative und keine exekutive Erfahrung verfüge, wie es der französische Präsident Emmanuel Macron mit Blick auf den EVP-Spitzenkandidaten Manfred Weber getan hat, ist ungehörig und grenzt fast schon an Infamie.

Die Verteidigung der „Hinterzimmer-Deals“ als demokratische Normalität, die Kritiker des Spitzenkandidatensystems ins Feld geführt haben, um die Nominierung der Nichtspitzenkandidatin Ursula von der Leyen für das Amt der Kommissionspräsidenten zu rechtfertigen, lenkt vom eigentlichen Problem ab. Wenn es darum geht, geeignete Kandidaten für die Spitzenpositionen zu finden und aufzustellen, sind solche Absprachen innerhalb der Parteienfamilien ebenso natürlich wie legitim. Dass die Staats- und Regierungschefs dabei ein gewichtiges Wort mitzureden haben, liegt ebenfalls auf der Hand, da es sich bei ihnen in der Regel zugleich um die Anführer der jeweiligen nationalen Parteien handelt. Entscheidend ist das, was nach der Wahl passiert. Hier haben die Wähler einen Anspruch darauf, dass die Kandidaten, die man ihnen vor der Wahl präsentiert hat, bei der Ämtervergabe tatsächlich zum Zuge kommen, sofern sie beziehungsweise ihre Parteien die notwendigen Mehrheiten erhalten. ${ }^{12}$

Ob das Spitzenkandidatensystem mit dem jetzigen Rückschlag schon endgültig gescheitert ist, bleibt abzuwarten. Die neue Kommissionspräsidentin von der Leyen hat dem Parlament versprochen, den Prozess beizubehalten und „zu verbessern“. Was das genau bedeutet, ist unklar. Im Kern besagt das Spitzenkandidatensystem ja zweierlei: Erstens soll nur der oder die Kommissionspräsident/in werden können, der/die zuvor als Spitzenkandidat/in für das Amt benannt wurde; und zweitens steht das Amt derjenigen Partei beziehungsweise Fraktion zu, die aus den Parlamentswahlen als „Sieger“ hervorgegangen ist und im EP über die meisten Mandate verfügt. Den ersten Teil als künftige Regel festzuschreiben, dürfte relativ unproblematisch sein. Das im zweiten Teil angesprochene Prinzip der „stärksten Partei“ weist dagegen gleich mehrere Fallstricke auf, die in vergleichbarer Form auch in vielen nationalstaatlichen parlamentarischen Demokratien vorzufinden sind.

Zunächst stellt sich die Frage, worauf das Stärkeverhältnis bezogen sein soll. Handelt es sich um die stärkste Partei/Fraktion des Parlaments, oder um die stärkste Kraft einer Mehrheit von Parteien/Fraktionen, die bereit wäre, einen bestimmten Kommissionspräsidenten zu wählen? Es ist ziemlich offensichtlich, dass für die Europäische Union nur das zuletzt genannte Prinzip gemeint sein kann, das auch der Praxis in den meisten parlamentarischen Demokratien mit Vielparteiensystemen entspricht. Der Europäische Rat würde wohl kaum eine Person für das Amt nominieren, bei der von vornherein feststeht, dass sie keine Aussichten hätte, eine Mehrheit für ihre Wahl im Parlament hinter sich zu versammeln.

Weniger einfach verhält es sich mit dem zweiten Prinzip - der stärksten Partei/Fraktion innerhalb einer Koalition. Dieses dürfte nur in einer Zweierkoalition weitgehend unstrittig sein, wo es immer einen Stärkeren gibt, oder wenn in einer Dreier- oder Viererkoalition die stärkste Fraktion über mehr Mandate verfügt als alle übrigen Koalitionspartner zusammen.

12 Vgl. Thomas Christiansen, EU-Spitzenkandidaten - neue Impulse und ihre Folgen für das politische System der EU, in: Integration, 38. Jg. (2015), H. 1, S. 26 - 43. 
2014 war ersteres der Fall: EVP und S\&D besaßen eine gemeinsame Mehrheit, so dass sie sich gegenseitig das Versprechen abnehmen konnten, dass dem jeweils stärkeren von ihnen das Amt des Kommissionspräsidenten zustehen würde - der Sozialdemokrat Martin Schulz hatte dabei gegenüber dem Christdemokraten Jean-Claude Juncker das Nachsehen. ${ }^{13} 2019$ traten dagegen die durch Emmanuel Macrons Partei La République en Marche gestärkten Liberalen als Dritte im Bunde hinzu, was in der Folge zu einer Blockade führte. Die Sozialdemokraten lehnten gemeinsam mit den Liberalen den Automatismus der stärksten Partei/ Fraktion ab, der dem EVP-Kandidaten Manfred Weber das Amt eingetragen hätte. Gleichzeitig waren sie aber auch nicht bereit, sich auf einen ihrer Kandidaten - Frans Timmermans oder Margarethe Vestager - zu verständigen. In diesem Fall hätte hinter dem/der Nominierten eine größere Mehrheit (34,9 Prozent der Sitze) gestanden als hinter Weber, dem Vertreter der stärksten Partei/Fraktion (24,2 Prozent).

\section{Direktwahl des Kommissionspräsidenten?}

Wenig spricht dafür, dass die Mehrheitsverhältnisse in Zukunft wieder einfacher werden der Trend in den Mitgliedstaaten weist eher in die gegenteilige Richtung einer weiteren Pluralisierung und Polarisierung. Damit verstärken sich die grundsätzlichen Zweifel an der Praktikabilität des Spitzenkandidatensystems. Doch wo liegen die Alternativen? Wenn die oben angedeutete These zutrifft, dass das Institutionensystem der EU von seiner Grundstruktur her größere Ähnlichkeiten mit dem gewaltentrennenden Präsidentialismus der USA aufweist als mit dem „gewaltenfusionierenden“ parlamentarischen System, das in Europa vorherrscht, wäre es konsequent, auch bei der Bestellung der Exekutive dem präsidentiellen Vorbild zu folgen. Statt über das Spitzenkandidatensystem eine stärkere Verkopplung von Parlaments- und Regierungswahl herbeizuführen, wäre dann der Kommissionspräsident in einem separaten Wahlakt zeitgleich mit dem Europäischen Parlament direkt zu wählen.

Wie hätte man sich das vorzustellen? In der heutigen Vielparteienstruktur würde vermutlich auch in einer Direktwahl keiner der Kandidaten in der Lage sein, auf Anhieb eine (absolute) Mehrheit zu erzielen. Bei Präsidentenwahlen auf nationaler Ebene wird in den meisten europäischen Ländern in dieser Situation üblicherweise eine Stichwahl zwischen den beiden bestplatzierten Bewerbern des ersten Wahlgangs fällig, was aber auf EU-Ebene angesichts von 420 Millionen Wahlbürgern kaum gangbar wäre. Um eine ausreichende

13 Weder die Wissenschaft noch die Öffentlichkeit (und auch nicht die betroffenen Akteure selbst, wie der Verfasser aus Gesprächen zu bezeugen weiß) haben dabei von dem Umstand Notiz genommen, dass die S\&D 2014 gemessen am absoluten Stimmenanteil ganz knapp vor der EVP lag (mit 24,7 zu 24,6 Prozent) - sie war daher die Hauptleidtragende der wahlsystembedingten ungleichen (parteipolitischen) Repräsentation, denn bei der Sitzverteilung kehrte sich das Verhältnis in 29,4 zu 25,4 Prozent zugunsten der EVP um. Auch 2019 blieben die Sozialdemokraten im Verhältnis von Stimmen- und Sitzanteil gegenüber der christdemokratischen Konkurrenz im Nachteil, wobei die EVP jetzt aber auch bei den absoluten Stimmen die Nase vorn hatte (20,7 zu 19,5 Prozent). Dem entsprach ein Sitzanteil von 24,2 zu 20,5 Prozent. Nach dem Ausscheiden der britischen Abgeordneten wird sich dieser Vorsprung noch etwas vergrößern (auf 22,8 zu 20,0 Prozent bei den Stimmen und 26,5 zu 20,3 Prozent bei den Sitzen). Für seine Hilfe bei der Ermittlung dieser Werte danke ich Manuel Müller. 
Legitimation jenseits der relativen Mehrheit zu besorgen, kämen folgende Varianten in Betracht: (1) ein Alternativstimmensystem, bei dem die Wähler Zweit- oder Drittpräferenzen angeben könnten; (2) ein indirektes Verfahren nach US-Vorbild - die Wahl erfolgt hier getrennt nach Mitgliedstaaten, denen eine vorab festgelegte Zahl von „Wahlmännern“ zusteht ${ }^{14}$; und (3) eine Verlegung der Stichwahl zwischen den beiden Bestplatzierten in das Europäische Parlament. (Dasselbe Verfahren könnte bei der zweiten Variante zum Zuge kommen, wenn ein Bewerber die absolute Mehrheit der Wahlmännerstimmen verfehlt.)

Die Volkswahl ${ }^{15}$ des Kommissionspräsidenten wäre ein radikaler Schritt, weil er den Europäischen Rat und - in der Variante 1 - auch das Parlament aus dem Prozess der Regierungsbestellung völlig ausschalten würde. Kritiker wenden deshalb ein, dass ein so scharfes mehrheitsdemokratisches Schwert zum konsensuellen Charakter der europäischen Politik nicht passe. ${ }^{16}$ Dem liegt ebenfalls ein doppelter Irrtum zugrunde: Erstens gibt es in jeder Demokratie, die diesen Namen verdient, so etwas wie ein mehrheitsdemokratisches Minimum. Die EU unterschreitet dieses Minimum bis heute nicht nur bei der Regierungsbestellung, sondern auch in den Bereichen beziehungsweise Zuständigkeiten, in denen die Vertreter der Mitgliedstaaten im Ministerrat und im Europäischen Rat einstimmig entscheiden. ${ }^{17}$

Zweitens - und noch wichtiger - bliebe ein mit größerer demokratischer Legitimation versehener Exekutivchef auf ein konsensuelles Zusammenwirken der an der Gesetzgebung beteiligten Organe angewiesen. Schon bei der Bestellung der Kommission müsste er auf das Einvernehmen mit dem Parlament und den mitgliedstaatlichen Regierungen achten. Dennoch hätte er das Vorrecht und die Bürde der politischen Initiative, könnte sich also nicht mehr hinter seinen Beamten oder den Vertretern des Ministerrats verstecken. Gleichzeitig wäre er derjenige, der die Einheit der Gemeinschaft institutionell verkörpert, ihre politischen Ziele nach innen und außen vertritt. Dies ließe sich auch durch die Zusammenlegung mit dem Amt des permanenten Ratspräsidenten deutlich machen, die - im Unterschied zur Direktwahl selbst - bereits im Rahmen der heutigen Verträge möglich wäre. Dies entspräche der „Doppelhut“-Lösung, die man für das Amt des Außenbeauftragten der EU gefunden hat.

Die starke Überlagerung der Europawahlen durch nationale Personen und Themen, an der das Spitzenkandidatensystem kaum etwas geändert hat, käme mit der Einführung der Direktwahl an ihr Ende. Nicht nur, dass die Wahl nach einem europaweit einheitlichen Verfahren durchgeführt werden müsste; auch der Kampf um die Stimmen selbst würde europäisiert: Die Parteien wären gezwungen, sich staatenübergreifend auf einen gemeinsamen Kandidaten zu einigen und mit einem personellen und programmatischen Gesamtangebot in die Wahl zu ziehen. Als Bewerber würden sich zum Beispiel amtierende oder ehe-

14 Um Sorgen der kleineren Länder vor einer Majorisierung zu zerstreuen, könnte sich die Verteilung der Wahlmännerstimmen dabei am Länderproporz im EP orientieren. Dies hätte allerdings den Nachteil, dass das demokratische Gleichheitsprinzip bei der Volkswahl des Kommissionspräsidenten genauso verletzt wäre wie bei einer ausschließlich parlamentarischen Bestellung, die den Befürwortern des Spitzenkandidatensystems vorschwebt.

15 Der im deutschen Sprachgebrauch übliche Begriff der Direktwahl übersieht, dass eine Volkswahl auch in einem indirekten Verfahren erfolgen kann.

16 Vgl. Marcus Höreth, Die Europäische Union im Legitimationstrilemma. Zur Rechtfertigung des Regierens jenseits der Staatlichkeit, Baden-Baden 1999, S. $272 \mathrm{ff.}$

17 Vgl. Frank Decker, a.a.O. (Fn. 11), S. $164-168$. 
malige Regierungschefs anbieten, die auch über die Grenzen ihres Heimatlandes hinaus Zugkraft hätten. Die Folge wäre ein höherer Mobilisierungsdruck, der das Zusammengehörigkeitsgefühl der Unionsbürger stärken, die Herausbildung eines europaweiten Parteiensystems unterstützen und sich natürlich auch auf die gleichzeitig stattfindenden Wahlen zum EP auswirken würde.

\section{Alternativen zum heutigen Bestellungsverfahren der Kommissare}

Um diesen gleichzeitigen Europäisierungs- und Demokratisierungseffekt zu erreichen, wären neben der Direktwahl allerdings mindestens zwei weitere Schritte notwendig. Erstens müsste - wie oben dargestellt - ein europaweites Wahlsystem geschaffen werden. Zweitens bräuchte es ein anderes Verfahren bei der Auswahl und Bestellung der Kommissare. Eine legitimatorische Aufwertung des Kommissionspräsidenten macht ja nur Sinn, wenn er zugleich über Mittel verfügt, die Positionen, für die er in der Wahl gestritten und ein Mandat bekommen hat, politisch umzusetzen. Dazu braucht es Kommissare an seiner Seite, die gleichgerichtete Ziele verfolgen und strukturell in der Lage sind, die entsprechenden Initiativen zu entwickeln.

So wie die Kommission heute ins Amt kommt und zusammengesetzt ist, lässt sich dies nicht gewährleisten. Einerseits beschränkt das Festhalten am gleichberechtigten Vertretungsanspruch aller 28 (oder demnächst: 27) Mitgliedstaaten ihre Arbeitsfähigkeit; das Gremium ist zu groß und die Abgrenzung der Ressorts wenig sachgerecht. Andererseits hat der Kommissionspräsident wenig Möglichkeiten, auf die personelle Auswahl der Kommissare Einfluss zu nehmen, da diese ausschließlich von den Regierungen der Mitgliedstaaten nominiert werden. Die Zusammenstellung der Kommission reflektiert insofern eher die nationalen Wahlergebnisse als das Ergebnis der Europawahlen. Lediglich über die Ressortzuteilung kann der Kommissionspräsident weitgehend selbst entscheiden.

Wie könnte man die beiden Probleme lösen? ${ }^{18}$ Für die Verkleinerung der Kommission würde es schon ausreichen, wenn die Länderparität etwas flexibler gehandhabt würde. Denkbar wäre zum Beispiel, dass man die Vertretung rotieren lässt oder sie unter den Mitgliedstaaten auslost. Dabei müsste den großen Ländern ein gewisser Vorrang eingeräumt werden. Gleichzeitig könnte man eine Abstufung nach Kommissaren und Vizekommissaren vornehmen, so dass jedem Land zumindest ein Stellvertreterposten sicher wäre. Bei der Bestellung der Kommissare sind ebenfalls verschiedene Varianten denkbar. Der Kommissionspräsident könnte etwa das Recht erhalten, einen Teil der Kommissare selbst zu nominieren, oder die Regierungen würden verpflichtet, mehrere Kandidaten vorzuschlagen, damit er aus einem größeren Pool auswählen kann. Vorstellbar wäre aber auch, das Nominierungsrecht von den Regierungen in die Hände der Wähler zu legen. Diese würden dann in der Europawahl zugleich über die jeweiligen nationalen Kandidaten für die Kommission entscheiden.

Das zuletzt genannte Verfahren könnte konkret wie folgt aussehen: In jedem Mitgliedstaat werden - je nach Größe - zwischen zwei und fünf Personen gewählt, die dem Kommissionspräsidenten als Kandidaten für die Kommission zur Auswahl stehen. Von den

18 Vgl. ders., Fallstricke der Parlamentarisierung. Warum das Bestellungsverfahren der EU-Kommissare der Reform bedarf, in: Recht und Politik, 50. Jg. (2014), H. 4, S. $199-203$. 
gewählten Kandidaten kämen nur etwa ein Drittel als Kommissare beziehungsweise Stellvertreter zum Zuge. Dies ist notwendig, weil der Kommissionspräsident ansonsten nicht über den nötigen Spielraum bei der Zusammenstellung seines Teams verfügte. Allerdings wäre es aus demokratischer Sicht kaum vermittelbar, wenn ausgerechnet die Kandidaten mit den besten Ergebnissen am Ende leer ausgingen - zumal wenn sie aus einem großen, bevölkerungsreichen Land stammen. Dieses Manko ließe sich durch eine Verkopplung mit der gleichzeitig stattfindenden Parlamentswahl beheben. Die Kandidaten für die Kommission würden danach im Normalfall zugleich für das EP kandidieren und die jeweiligen Listen der Parteien in ihren Ländern anführen. Werden sie gewählt, wäre ihnen in jedem Fall ein Sitz im Parlament sicher.

Eine solche Wahl würde die Kommission demokratisch weiter aufwerten und den Europäisierungseffekt verstärken. Es gäbe nicht nur einen zusätzlichen Anreiz, sich an den Wahlen zu beteiligen. Auch den Parteien fiele es vermutlich leichter, für einen europäischen Spitzenkandidaten aus einem anderen Land zu werben, wenn diesem ein Kandidat aus dem eigenen Land zur Seite stünde. Die nationale Orientierung der Wähler, die sich allein schon aufgrund der Sprachbarrieren ergibt, würde auf diese Weise für die europäischen Zwecke „eingespannt“. Die Anwärter auf das Amt des Kommissionspräsidenten hätten wiederum den Vorteil, dass sie schon im Vorfeld der Wahl in ihrer Parteienfamilie auf die Aufstellung geeigneter Bewerber hinwirken könnten, mit denen sie die spätere Kommissionsmannschaft bilden. Dies würde auch den Zusammenschluss der bisher nur locker verbundenen nationalen Parteiorganisationen zu wirklichen europäischen Parteien befördern.

Ein so verändertes Bestellungsverfahren der Kommissare hätte zugleich den positiven Nebeneffekt, die langwierigen und bisweilen von sachfremden Motiven überlagerten Anhörungen der Kommissarsanwärter im Europäischen Parlament entbehrlich zu machen. Zusammen mit der Bestätigung der gesamten Kommission durch das EP sollen diese im derzeitigen System einen Ausgleich dafür schaffen, dass die Abgeordneten bei der Installierung der europäischen Regierung gegenüber den mitgliedstaatlichen Regierungen bisher nur die „zweite Geige“ spielen. Dass das EP mindestens einen der vorgeschlagenen Kommissare zu Fall bringt ${ }^{19}$, gehört inzwischen zu den fest institutionalisierten Ritualen der europäischen Politik, die man eher unter der Rubrik „Folklore“ denn als tatsächliche Machtentfaltung verbuchen sollte. Dasselbe gilt für die Möglichkeit einer Ablehnung der gesamten Kommission am Ende des Verfahrens, deren Drohwirkung mangels Glaubwürdigkeit sehr überschaubar sein dürfte.

\section{Reformen mithilfe unterschiedlicher Geschwindigkeiten?}

Bleibt die Frage nach den Realisierungschancen. Die Direktwahl des Kommissionspräsidenten ist keine exotische Idee von Außenseitern - immerhin steht sie bei der CDU seit 2011 im Parteiprogramm. Im Ringen um die Nominierung des Kommissionspräsidenten hat Angela Merkel sie als denkbare Option jetzt erneut ins Spiel gebracht. Umsetzbar wäre

192019 musste unter anderem die für das Binnenmarktressort nominierte französische Kandidatin Sylvie Goulard „daran glauben“, deren Ablehnung vor allem von der EVP-Fraktion betrieben wurde. Dass es sich dabei um eine Retourkutsche gegen Präsident Macron handelte, der zuvor EVP-Spitzenkandidat Weber als Kommissionschef verhindert hatte, war ziemlich offensichtlich. 
der Vorschlag nur mit einer Vertragsänderung, die einstimmig erfolgen muss und deshalb auf absehbare Zeit unerreichbar erscheint. Um eine neue Verfassungsdebatte dürfte die EU mittel- und langfristig aber bereits wegen des Einstimmigkeitsprinzips nicht umhinkommen, das demokratisch fragwürdig ist und ihre Handlungsfähigkeit auf zentralen Feldern bedroht. Auch andere demokratische Mängel wie der Verstoß gegen die Stimmrechtsgleichheit oder das dem Parlament bis heute vorenthaltene Initiativrecht würden, wenn man sie beseitigen will, Vertragsänderungen notwendig machen.

Anders sieht es bei den Vorschlägen aus, die sich unterhalb der Vertragsebene bewegen, zum Beispiel das Bestellungsverfahren der Kommissare. Hier könnten integrationswillige Staaten, die der Demokratisierung aufgeschlossen gegenüberstehen, mit eigenen Reformen vorauseilen und die anderen irgendwann mitziehen. Warum sollte ein Europa der unterschiedlichen Geschwindigkeiten nicht auch in institutioneller Hinsicht möglich sein? Über diese und andere Fragen der demokratischen Weiterentwicklung der EU brauchen wir dringend einen Ideenwettbewerb. Dass man dabei nicht primär auf die Staats- und Regierungschefs setzen sollte, zeigt ihr Umgang mit dem Spitzenkandidatensystem. Die Impulse müssen vielmehr vom jetzt neu gewählten Parlament und einer sich zunehmend formierenden europäischen Öffentlichkeit ausgehen. 